Pacific Journal of Mathematics

LATTICES OF LOWER SEMI-CONTINUOUS FUNCTIONS AND

LouIS D. NEL 


\title{
LATTICES OF LOWER SEMI-CONTINUOUS FUNCTIONS AND ASSOCIATED TOPOLOGICAL SPACES
}

\author{
LOUIS D. NEL
}

\begin{abstract}
In this paper the lattice of all real-valued lower semi-continuous functions on a topological space is studied. It is first shown that there is no essential loss if attention is restricted to $T_{0}$-spaces. By suitably topologizing a certain set of equivalence classes of prime ideals, it is shown that a topological space is determined by the lattice. This topological space is homeomorphic with the original space $X$ whenever $X$ has the property that every non-empty irreducible closed set is a point closure. The sublattices of functions taking values only in intervals of the form $(a, b]$ and $[a, b]$ are compared. Relations between the above function lattices and the lattice of all closed subsets are also discussed.
\end{abstract}

Preliminaries. Let $L_{R}(X)$ (or briefly $L_{R}$ ) denote the lattice of all lower semi-continuous functions defined on the topological space $X$ into the real line $R$. It is well known that $L_{R}$ is a conditionally complete distributive lattice under the usual order relation $f \leqq g$ which means $f(x) \leqq g(x)$ for all $x \in X$ (except where otherwise indicated, latticetheoretic terminology will follow [1]). For an arbitrary bounded nonempty set $F \subset L_{R}$ the join $\bigvee F$ satisfies $\bigvee F(x)=\sup \{f(x): f \in F\}$; the meet $\Lambda F$ is defined as $\bigvee\left\{g \in L_{R}: g \leqq f\right.$ for all $\left.f \in F\right\}$ and it should be noted that $\Lambda F(x)=\inf \{f(x): f \in F\}$ need not hold when $F$ is infinite. The constant function with value $s$ will be written $s$.

The elements of $L_{R}$ can be regarded more conveniently as continuous functions on $X$ into $R_{l}$ where $R_{l}$ is the $T_{0}$-space obtained by giving the real line the topology having as non-empty closed sets those of the form $\{x: x \leqq r\}(r \in R)$.

Some other function lattices will also be considered towards the end of the paper. Let $H, I$ denote the real intervals $(0,1],[0,1]$ respectively and $L_{H}(X), L_{I}(X)$ the sublattices of $L_{R}(X)$ consisting of those functions which take values only in $H, I$ respectively (no essential difference will arise if any extended real intervals $(a, b],[a, b]$ are taken for $H, I$ ).

We will use $\mathscr{C}(X)$ to denote the lattice of closed subsets of the topological space $X$. The set of nonzero irreducible elements of $\mathscr{C}$ will be denoted by $\mathscr{A}(X)$; thus $\mathscr{A}$ consists of the nonempty closed sets $A$ which cannot be expressed as the union of two properly smaller closed sets. Closures will be written $\operatorname{cl} A$ with $\operatorname{cl} x=\operatorname{cl}\{x\}$ for point closures. 
Relations between $X$ and the lattice $\mathscr{C}(X)$ have been studied by several authors notably Thron [3] and Blanksma [2]. We now give a summary of relevant facts from these two papers in a form suitable for our needs. We restrict attention to $T_{0}$-spaces as this entails no essential loss of generality.

The set $\mathscr{A}$ can be topologized by taking as closed sets those of the form $\{A \in \mathscr{A}: A \subset F\}$ where $F \in \mathscr{C}$ (see [3, proof of 3.1] and [2, I, ch 2]. We will denote the topological space thus obtained by $\pi X$. Since every point closure $\mathrm{cl} x$ is irreducible, the mapping $\gamma(x)=\mathrm{cl} x$ is an embedding of $X$ into the set $\mathscr{A}$ and moreover it is a topological embedding of $X$ into $\pi X$ (see [2, I, 3.4]). An important class of spaces are those in which every $A \in \mathscr{A}$ is a point closure (see [2, I, 2.2]). Such spaces will be called pc-spaces; $T_{D}$-spaces (see [3]) are defined to be those for which $\mathrm{cl} x-\{x\}$ is always a closed set. It is perhaps worth pointing out that these two types of spaces can be regarded as the extreme cases of a certain situation. If we use $\mathscr{A}^{*}$ to denote the set of strongly irreducible elements of $\mathscr{A}$ (i.e. those $A \in \mathscr{A}$ which cannot be written $A=\operatorname{clU}_{s \in S} B_{s}$ for any family $\left(B_{s}\right) \subset \mathscr{C}$ with $B_{s} \varsubsetneqq$ $A$ ), then we have for any $T_{0}$-space $X$

$$
\mathscr{A}^{*} \subset \gamma(X) \subset \mathscr{A} \text {. }
$$

(It is easily verified that each $A \in \mathscr{A}^{*}$ must be a point closure). The $T_{D}$-spaces can now be described as those for which $\mathscr{A}^{*}=\gamma(X)$ while the point closure spaces are those for which $\gamma(X)=\mathscr{A}$. The specific results concerning $\pi X$ and $\mathscr{C}$ which will be needed in this paper can now be stated as follows. (When we say $X$ is determined as a space with property $P$ by the lattice $C(X)$ (resp. $L_{R}(X)$ ) we mean that if $Y$ is also a space with property $P$ then $X$ and $Y$ are homeomorphic iff $C(X)$ and $\mathscr{C}(Y)$ (resp. $L_{R}(X), L_{R}(Y)$ ) are isomorphic.)

\section{Known facts.}

For any $T_{0}$-space $X$ we have

(a) $\pi X$ is a pc-space.

(b) If $X$ is a pc-space, then $X$ and $\pi X$ are homeomorphic.

(c) Every $f \in L_{H}(X)$ has a unique extension $f^{\pi} \in L_{H}(\pi X)$ (here we have identified $X$ with a dense subspace of $\pi X$, as may be done).

(d) The lattices $\mathscr{C}(X)$ and $\mathscr{C}(\pi X)$ are isomorphic.

(e) The space $\pi X$ is determined as a pc-space by the lattice $\mathscr{C}(X)$ (hence if $X$ is a pc-space, it is determined as such by the lattice $\mathscr{C}(X)$ ).

(f) If $X$ is a $T_{D}$-space, it is determined as such by the lattice $\mathscr{C}(X)$.

For (a) through (e), see [2, I, chapters 2,3]; (c) is not stated explicitly, but $\mathrm{H}$. Herrlich has pointed out in his review of [2] (MR 
$37,5851)$ that the $p c$-spaces form an epireflective subcategory of the $T_{0}$-spaces and (c) follows at once from the fact that $\pi X$ is the epireflection of $X$. See [3] for a proof of $(f)$.

THEOREM 2. Let $T$ be any topological space and $X$ its $T_{0}$-identification. Then the lattices $L_{R}(T)$ and $L_{R}(X)$ are isomorphic.

Proof. $X$ is the quotient space $T / \rho$, where the relation $x \rho y$ means $\operatorname{cl} x=\operatorname{cl} y$. Let $c$ denote the canonical mapping of $T$ onto $T / \rho$. Notice that $\operatorname{cl} x \neq \operatorname{cl} y$ iff $f(x) \neq f(y)$ for some $f \in L_{R}(T)$. Hence for each $f \in L_{R}(T)$ there is a unique function $f^{*}$ on $T / \rho$ such that $f^{*} \circ c=f$. Since $f^{*}$ is defined on a quotient space, its continuity follows from the continuity of $f$. The proof is completed with the simple verification that $f \rightarrow f^{*}$ is an isomorphism of $L_{R}(T)$ onto $L_{R}(T / \rho)$.

In view of this theorem all spaces $X$ under discussion will from now on supposed to be $T_{0}$-spaces.

Closed prime ideals in $L_{R}(X)$. By an ideal in $L_{R}$ will be meant a nonempty proper subset $J$ of $L_{R}$ such that $f \wedge g \in J$ whenever $f \in$ $J, g \in L_{R}$ and $f \vee g \in J$ whenever $f, g \in J$ (here we differ from [1] where an ideal in a lattice need not be a proper subset). An ideal $J$ will be called closed if for any $G \subset J$ such that $\bigvee G$ exists in $L_{R}$ we have $\mathrm{V} G \in J$. As usual, prime ideal will mean an ideal which contains $f \wedge g$ only if it contains $f$ or $g$.

Proposition 3. The set $I(r, A)=\left\{f \in L_{R}(X): f(x) \leqq r\right.$ when $x \in$ $A\}$ is a closed prime ideal, where $r \in R$ and $A \in \mathscr{A}$. Every closed prime ideal in $L_{R}$ is of this form.

Proof. If $f_{1} \wedge f_{2} \in I(r, A)$, then the closed sets $A_{i}=\left\{x \in A: f_{i}(x) \leqq\right.$ $r\}(i=1,2)$ have $A$ as their union. Since $A$ is irreducible we conclude that $A=A_{i}$ and $f_{i}(x) \leqq r$ when $x \in A$ for some $i$. Hence $I(r, A)$, which is clearly a closed ideal, is prime. Let us now consider any closed prime ideal $P$ in $L_{R}$ and let $B$ denote the set of all $x \in X$ for which the number

$$
m(x)=\sup \{p(x): p \in P\}
$$

exists. We show that $m(x)$ is the same number for all $x \in B$. If $m(y)<m(z)$ holds we can chose $s, t \in R$ such that

$$
m(y)<s<m(z)<t .
$$

Given any $g \in P$ we define elements $u, v \in L_{R}$ as follows: $u=s \vee g$ and 


$$
v(x)=\left\{\begin{array}{l}
g(x) \text { when } g(x) \leqq s \\
t \vee g(x) \text { when } g(x)>s .
\end{array}\right.
$$

Then $g=u \wedge v, u \notin P$ and $v \notin P$ which is absurd. We conclude that $B$ is of the form $\{x \in X: m(x)=r\}$ for some $r \in R$. The function $e=$ $\mathrm{V}\{p \wedge s: p \in P\}$ belongs to $P$ (where $r<s$ ) and so $\{x: e(x) \leqq r\}=B$ is a closed set. Moreover, $B$ is irreducible for if it is the union of closed proper subsets $B_{1}, B_{2}$ then

$$
f_{i}(x)=\left\{\begin{array}{l}
r \text { if } x \in B_{i} \\
s \text { if } x \notin B_{i}
\end{array} \quad(i=1,2)\right.
$$

satisfy $f_{1} \wedge f_{2}=e \in P, f_{1} \notin P, f_{2} \notin P$. Finally, if $f \in I(r, B)$, then $f=$ $\mathrm{V}\{f \wedge p: p \in P\} \in P$ and we conclude $P=I(r, B)$.

The symbol $\mathscr{C}$ will be used from now on to denote the set of closed prime ideals in $L_{R}$. For a given $P \in \mathscr{K}$ the irreducible set $A$ such that $P=I(r, A)$ will be called the carrier of $P$ and for elements $P, Q \in \mathscr{C}$ we define $P \sim Q$ to hold iff $P$ and $Q$ have the same carrier. The relation thus defined is evidently an equivalence relation and it will be of importance to know that this relation can be characterized in terms of the lattice structure of $L_{R}(X)$ without reference to $X$. For this purpose we make the following definition. An ideal $I(r, A) \in$ $\mathscr{K}^{-}$is called quasi-minimal if $\{P \in K: P \subset I(r, A)\}$ forms a chain under the relation $\subset$.

LeMma 4.

(a) An ideal $I(r, A) \in \mathscr{K}$ is quasi-minimal iff $A$ is maximal irreducible.

(b) For quasi-minimal ideals the relation $I(r, A) \subset I(s, B)$ holds iff $r \leqq s$ and $A=B$.

Proof. The elements $P \in \mathscr{C}$ with $P \subset I(r, A)$ are those of the form $I\left(r^{\prime}, B\right)$ where $r^{\prime} \leqq r$ and $B \supset A$. These elements form a chain iff $B=A$ holds. Thus (a) follows and (b) is an immediate consequence.

Notice that $P \sim Q$ can hold only if $P \subset Q$ or $Q \subset P$. So in order to obtain the desired characterization of the relation $\sim$ it is enough to consider comparable ideals.

Lemma 5. Let $P, Q \in \mathscr{C}$ satisfy $P \subset Q$. Then $P \sim Q$ holds iff there exists a pair of quasi-minimal ideals $J_{1}, J_{2} \in \mathscr{C}$ such that

$$
J_{1} \subset J_{2}, J_{1} \subset P \cap J_{2} \text { and } P \vee J_{2}=Q,
$$

where $P \vee J_{2}=\left\{f \vee g: f \in P, g \in J_{2}\right\}$.

Proof. If $P \sim Q$ with $P \subset Q$, then $P=I(r, A), Q=I(s, A)$ for 
some $r<s$. A standard application of Zorn's lemma shows that there is a maximal set $M \in \mathscr{A}$ such that $M \supset A$. Put $J_{1}=I(r, M), J_{2}=$ $I(s, M)$. It is easy to verify that $J_{1}, J_{2}$ satisfy the requirements of the lemma. Conversely, let us start with $P=I(r, A) \subset I(s, B)=Q$ and $J_{1}=I(m, M), J_{2}=I(n, N)$ as stated. From $J_{1} \subset P$ and $P \vee J_{2}=Q$ we deduce respectively $M \supset A$ and $A \cap N=B$. Using $J_{1} \subset J_{2}$ and Lemma 4 we conclude that $M=N$ and hence $A=B$. This completes the proof.

In view of Lemma 5 and the remark preceding it we have the following important fact.

CoRollary. The set $\Omega\left(L_{R}\right)$ (or briefly $\Omega$ ) of equivalence classes $\omega(P)=\{Q: P \sim Q\}(P \in \mathscr{K})$ is determined by the lattice structure of $L_{R}$.

The topological space $\Omega\left(L_{R}\right)$. Our next undertaking is to introduce a topology in the set $\Omega$. We do this by specifying a subset $\Sigma \subset \Omega$ to be closed iff it has the following property: if $P, Q_{t} \in \mathscr{K} \quad(t \in T)$ are such that $P \supset \bigcap_{t \in T} Q_{t} \neq \varnothing$ holds where each $Q_{t}$ belongs to some $\sigma \in \Sigma$, then $\omega(P) \in \Sigma$. This is reminiscent of the hull-kernel topology encountered in commutative ring theory.

Theorem 6. The topological space $\Omega\left(L_{R}\right)$ is determined by the lattice structure of $L_{R}(X)$. It is a pc-space, homeomorphic to the space $\pi X$.

Proof. It will be shown that $\Omega$ can be put in a $1-1$ correspondence with the $p c$-space $\pi X$ in such a way that the sets called closed above correspond to the closed subsets of $\pi X$. We note first of all that, by definition, a class $\omega(P) \in \Omega$ consists of all ideals $Q \in \mathscr{K}$ which have a common carrier. Thus by putting

$$
I(A)=\{I(r, A): r \in R\}
$$

we obtain a $1-1$ correspondence between the elements $A \in \pi X$ and the elements $I(A) \in \Omega$. Let us now consider an arbitrary subset $\Sigma=$ $\{I(A): A \in \mathscr{S}\}(\mathscr{S} \subset \pi X)$ of $\Omega$ and any $I(s, B) \in \mathscr{K}$. Then $I(s, B) \supset \bigcap_{t \in T} I\left(r_{t}\right.$, $\left.A_{t}\right) \neq \varnothing$ holds for some family $\left\{I\left(r_{t}, A_{t}\right): t \in T\right\}$ with all $A_{t} \in \mathscr{S}$ iff $B \subset \mathrm{cl} \cup \mathscr{S}$. Indeed, if $B \not \subset \mathrm{cl} \cup \mathscr{S}$ and $f \in \cap_{t \in T} I\left(r_{t}, A_{t}\right)$ holds with all $A_{t} \in \mathscr{S}$, then either $f \notin I(s, B)$ or any function $g$ such that $g(x)=$ $s^{\prime} \vee f(x)\left(s^{\prime}>s\right)$ when $x \in X \mid \operatorname{cl}_{t \in T} A_{t}$ and which agrees with $f$ on $\mathrm{cl}_{t \in T} A_{t}$ satisfies $g \in \bigcap_{t} I\left(r_{t}, A_{t}\right)$ and $g \notin I(s, B)$; and if $B \subset \operatorname{cl} \cup \mathscr{S}$ holds then $I(s, B) \supset \cap\{I(s, A): A \in \mathscr{S}\} \neq \varnothing$ clearly holds. We conclude that $\Sigma$ is closed in $\Omega$ iff it is the image of some closed set $\mathscr{S} \subset \pi X$ under the mapping $A \rightarrow I(A)$. Hence $\Omega$ is homeomorphic to $\pi X$. That the topological space $\Omega$ is determined by the lattice $L_{R}$ is clear from the corollary to Lemma 5 and the definition of closed sets in $\Omega$ : only 
the lattice structure of $L_{R}$ is used to define it.

CoROllary 7. If $X$ is a pc-space, then $X$ is homeomorphic to $\Omega\left(L_{R}\right)$ and is therefore determined by the lattice structure of $L_{R}(X)$.

This follows at once in view of Theorem 6 and the known fact $1(b)$.

Remarks on the lattice $L_{H}(X)$. We will now turn our attention to the sublattice $L_{H}(X)$ of $L_{R}(X)$. The results proved above for $L_{R}(X)$ all remain valid if $L_{H}$ is substituted for $L_{R}$ and some minor adaptations are made, the most important of which is to restrict the variable $r$ in the ideals $I(r, A)$ to the interval $0<r<1$. As a matter of fact, the theory for $L_{H}(X)$ can be simplified by using prime elements (i.e. those $g \in L_{H}$ such that $g<1$ and $g=u \wedge v$ only if $g=u$ or $g=v$ ) rather than closed prime ideals. This will be clear from the following fact.

\section{Proposition 8.}

(a) $g \in L_{H}$ is prime iff $g$ is of the form $e_{r A}$ where $e_{r A}(x)=r$ when $x \in A$ and $e_{r_{A}}(x)=1$ when $x \in X \backslash A(0<r<1$ and $A \in \mathscr{A})$.

(b) Every closed prime ideal in $L_{H}$ is of the form $\left\{f: f \leqq e_{r A}\right\}$.

The proof is very much like that of Proposition 3 and is therefore omitted.

By using equivalence classes of primes in $L_{H}$ one can now prove as for $L_{R}$ that the lattice $L_{H}$ determines a topological space $\Omega\left(L_{H}\right)$ which is homeomorphic to $\pi X$ and hence to $X$ whenever $X$ is a $p c$-space.

We now consider some properties of $L_{I I}$ which are not included in the theory presented for $L_{R}$.

\section{Proposition 9.}

(a) The lattices $L_{H}(X)$ and $L_{H}(\pi X)$ are isomorphic.

(b) For a given $X$ the lattices $L(X)$ and $\mathscr{C}(X)$ determine each other.

(c) If $X$ is a $T_{D}$-space, then it is determined as such by the lattice $L_{H}(X)$.

Proofs. (a) The mapping $f \rightarrow f^{\pi}$ (see $1(\mathrm{c})$ ) is easily seen to be an isomorphism of $L_{H}(X)$ onto $L_{H}(\pi X)$.

(b) If the lattice $L_{H}(X)(\operatorname{resp} . \mathscr{C}(X))$ is known then $\pi X$ is known and thus also the lattice $\mathscr{C}(\pi X)=\mathscr{C}(X)$ (resp. $\left.L_{H}(\pi X)=L_{H}(X)\right)$.

(c) The lattice $L_{H}(X)$ determines the lattice $\mathscr{C}(X)$ which determines $X$ as a $T_{D}$-space.

Let us give an example to show that 9 (a) is not valid for $L_{R}(X)$. If $X$ is the subspace $(0,1)$ of $R_{l}$, then $\pi X$ is the subspace $(0,1]$ of $R_{l}$. In the case of both $X$ and $\pi X$ there is just one maximal irreducible 
set namely the whole space. Thus the quasi-minimal ideals in both $L_{R}(X)$ and $L_{R}(\pi X)$ are the principal ideals $\{f: f \leqq r\}$ of the constant functions (see Lemma 4(a)). Now every $f$ in $L_{R}(\pi X)$ attains a maximum value $f(1)$ and so every $f \in L_{R}(\pi X)$ belongs to some quasi-minimal ideal. This cannot hold for $L_{R}(X)$ which clearly contains functions unbounded above. The two lattices are therefore not isomorphic.

There is in fact a class wider than the $p c$-spaces such that the lattice $L_{R}(X)$ determines $X$ whenever $X$ belongs to this class. This will be discussed in a later paper. L. D. Nel and R. G. Wilson, Epireflections in the category of $T_{0}$-spaces (to appear in Fund. Math.).

Remarks on the lattice $L_{I}(X)$. The method used above to prove that the lattices $L_{R}(X)$ and $L_{H}(X)$ determine $p c$-spaces cannot be applied to $L_{I}(X)$. Theorem 2, Propositions 3 and 8 remain valid for $L_{I}(X)$ but Lemma 4(a) fails (and therefore all further results based on it). It fails because in the case of $L_{I}(X)$ there are two types of quasi-minimal ideals, namely those of the form $I(0, A)$ for non-maximal $A \in \mathscr{A}$ and those of the form $I(r, A)$ for $0<r<1$ and maximal $A \in$ $\mathscr{A}$. There appears to be no lattice theoretic method of distinguishing between these types.

It seems plausible nevertheless that $L_{I}(X)$ should determine $X$ whenever it is a $p c$-space. A settlement of this open question should be interesting.

\section{REFERENCES}

1. Garret Birkhoff, Lattice Theory, Amer. Math. Soc., Colloquium Publications 25, Providence, 1967.

2. T. Blanksma, Lattice characterizations of topologies and compactifications, Doctoral dissertation, Rijks universiteit te Utrecht, 1968.

3. W. J. Thron, Lattice-equivalence of topological spaces, Duke Math. J., 29 (1962), 671-679.

Received February 11, 1971. This research was supported partly by the National Research Council of Canada (grant A5297).

Carleton University, Canada 



\section{PACIFIC JOURNAL OF MATHEMATICS}

\section{EDITORS}

\section{H. SAMELSON}

Stanford University

Stanford, California 94305

\section{R. HOBBY}

University of Washington Seattle, Washington 98105

\section{J. DugundJI}

Department of Mathematics University of Southern California Los Angeles, California 90007

RICHARD ARENS

University of California Los Angeles, California 90024

\section{ASSOCIATE EDITORS}
E. F. BECKENBACH
B. H. Neumann
F. WoLF
K. YoSHIDA

\section{SUPPORTING INSTITUTIONS}

UNIVERSITY OF BRITISH COLUMBIA CALIFORNIA INSTITUTE OF TECHNOLOGY

UNIVERSITY OF CALIFORNIA

MONTANA STATE UNIVERSITY

UNIVERSITY OF NEVADA

NEW MEXICO STATE UNIVERSITY

OREGON STATE UNIVERSITY

UNIVERSITY OF OREGON

OSAKA UNIVERSITY

\author{
UNIVERSITY OF SOUTHERN CALIFORNIA \\ STANFORD UNIVERSITY \\ UNIVERSITY OF TOKYO \\ UNIVERSITY OF UTAH \\ WASHINGTON STATE UNIVERSITY \\ UNIVERSITY OF WASHINGTON \\ * * * * \\ AMERICAN MATHEMATICAL SOCIETY \\ NAVAL WEAPONS CENTER
}

The Supporting Institutions listed above contribute to the cost of publication of this Journal, but they are not owners or publishers and have no responsibility for its content or policies.

Mathematical papers intended for publication in the Pacific Journal of Mathematics should be in typed form or offset-reproduced, (not dittoed), double spaced with large margins. Underline Greek letters in red, German in green, and script in blue. The first paragraph or two must be capable of being used separately as a synopsis of the entire paper. The editorial "we" must not be used in the synopsis, and items of the bibliography should not be cited there unless absolutely necessary, in which case they must be identified by author and Journal, rather than by item number. Manuscripts, in duplicate if possible, may be sent to any one of the four editors. Please classify according to the scheme of Math. Rev. Index. to Vol. 39. All other communications to the editors should be addressed to the managing editor, Richard Arens, University of California, Los Angeles, California, 90024.

50 reprints are provided free for each article; additional copies may be obtained at cost in multiples of 50 .

The Pacific Journal of Mathematics is published monthly. Effective with Volume 16 the price per volume (3 numbers) is $\$ 8.00$; single issues, $\$ 3.00$. Special price for current issues to individual faculty members of supporting institutions and to individual members of the American Mathematical Society: $\$ 4.00$ per volume; single issues $\$ 1.50$. Back numbers are available.

Subscriptions, orders for back numbers, and changes of address should be sent to Pacific Journal of Mathematics, 103 Highland Boulevard, Berkeley, California, 94708.

PUBLISHED BY PACIFIC JOURNAL OF MATHEMATICS, A NON-PROFIT CORPORATION

Printed at Kokusai Bunken Insatsusha (International Academic Printing Co., Ltd.), 270, 3-chome Totsuka-cho, Shinjuku-ku, Tokyo 160, Japan. 


\section{Pacific Journal of Mathematics}

\section{Vol. 40, No. $3 \quad$ November, 1972}

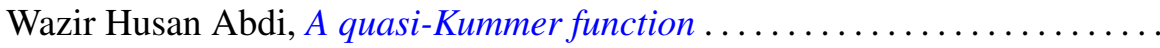

Vasily Cateforis, Minimal injective cogenerators for the class of modules of

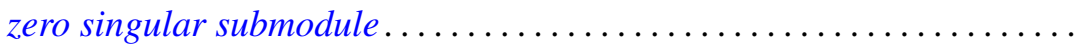

W. Wistar (William) Comfort and Anthony Wood Hager, Cardinality of

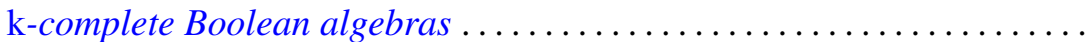

Richard Brian Darst and Gene Allen DeBoth, Norm convergence of martingales of Radon-Nikodym derivatives given a $\sigma$-lattice ..........

M. Edelstein and Anthony Charles Thompson, Some results on nearest points and support properties of convex sets in $c_{0} \ldots \ldots \ldots \ldots \ldots$

Richard Goodrick, Two bridge knots are alternating knots .

Jean-Pierre Gossez and Enrique José Lami Dozo, Some geometric properties related to the fixed point theory for nonexpansive mappings ..........

Dang Xuan Hong, Covering relations among lattice varieties .............

Carl Groos Jockusch, Jr. and Robert Irving Soare, Degrees of members of $\Pi_{1}^{0}$

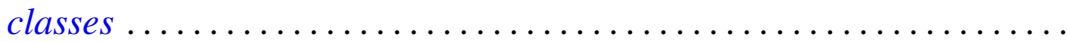

565

575

605

Leroy Milton Kelly and R. Rottenberg, Simple points in pseudoline

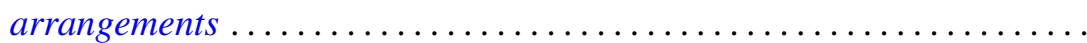

Joe Eckley Kirk, Jr., The uniformizing function for a class of Riemann surfaces....

Glenn Richard Luecke, Operators satisfying condition $\left(G_{1}\right)$ locally ... 629

T. S. Motzkin, On L $(S)$-tuples and l-pairs of matrices ... . .

Charles Estep Murley, The classification of certain classes of torsion free

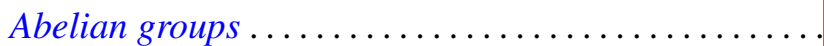

Louis D. Nel, Lattices of lower semi-continuous functions and associated topological spaces.

David Emroy Penney, II, Establishing isomorphism between tame prime

knots in $E^{3}$. . .

Daniel Rider, Functions which operate on $\mathscr{F} L_{p}(T), 1<p<2$

Thomas Stephen Shores, Injective modules over duo rings ...

Stephen Simons, A convergence theorem with boundary. .

703

Stephen Simons, Maximinimax, minimax, and antiminimax theorems and a

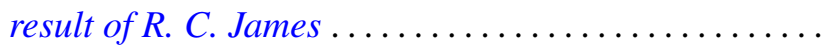

Stephen Simons, On Ptak's combinatorial lemma ........

Stuart A. Steinberg, Finitely-valued $f$-modules............

Pui-kei Wong, Integral inequalities of Wirtinger-type and fourth-order

elliptic differential inequalities .

Yen-Yi Wu, Completions of Boolean algebras with partially additive

operators ..................................

Phillip Lee Zenor, On spaces with regular $G_{\delta}$-diagonals . . . 